\title{
Design and performance analysis of a PV solar powered water pumping system
}

\author{
H. Jerbi*
}

College of Engineering, University of Hail, Hail, Saudi Arabia

\section{ARTICLE INFO}

\section{Article history:}

Received 14 November 2016

Received in revised form

15 April 2017

Accepted 24 April 2017

\section{Keywords:}

MPPT

PV generator

DC motor pump Gain scheduling

control

Feedback linearization

Discrete-time

Nonlinear systems

\section{Introduction}

The growing demand for energy and the pollution resulting from the use of fossil fuels has recently prompted the search for an alternative renewable energy. In this context, photovoltaic energy has become one of the major renewable energy sources and a possible remedy to energy production problems. Photovoltaic system (PV) technologies are growing rapidly and therefore play a vital role in electrical technologies. They are deemed to be a green energy for the on-going century. A great deal of current work focuses on cells and PV systems to improve the electrical performance of the $\mathrm{PV}$ panels and reduce energy losses within the PV systems (Salas et al., 2006; Mrabti et al., 2010). This will considerably reduce the cost of PV systems and encourage consumers to use this green energy. Photovoltaic cell provides a maximum power point (MPP) that varies according to the sun's irradiance variation and temperature (Mrabti et al., 2010). This is achieved through analogue or digital Maximum Power Point Tracking (MPPT) controls (Salameh et al., 1991; El Ouariachi et al., 2009). A significant number of MPPT control systems have been developed, starting with simple techniques, such as voltage or current variations (Bacha et al., 2008). The current MPPT control is

\section{* Corresponding Author.}

Email Address: h.jerbi@uoh.edu.sa

https://doi.org/10.21833/ijaas.2017.05.022

2313-626X/C 2017 The Authors. Published by IASE.

This is an open access article under the CC BY-NC-ND license

(http://creativecommons.org/licenses/by-nc-nd/4.0/) proposed in (El Ouariachi et al., 2009; Esram and Chapman, 2007). In the case of the analogue MPPT control, there is a problem of congestion divergence of the electronic components used (Shraif, 2002; El Ouariachi et al., 2009). However, in the case of the digital MPPT control, many algorithms have been used in the literature (Hussein et al., 1995; Salas et al., 2006; Esram and Chapman, 2007), and include the incremental conductance method IC, (Cocconi and Rippel, 1990; Veerachary et al., 2003) the fuzzy logic method (Bahgat et al., 2005), the neural networks method (Swrup and Ansari, 2012) and the traditional perturbation and observation method, which poses precision problems on the regulation around the MPPT (El Ouariachi et al., 2009).

Regarding PV systems, the problems that arise include how to optimize the power supplied by the PV panels (Bacha et al., 2008; Charfeddine et al., $2012 \mathrm{~b}$ ), how to transfer to the load, how to control charge and discharge (Veerachary et al., 2003), and how to track the sun by PV (El Ouariachi et al., 2009; Mrabti et al., 2010).

For that reason, a nonlinear gain scheduling control is investigated to control the duty ratio in such a manner that enables finding and tracking the Maximum Power Point," so that the maximum power is continuously achieved.

In this work, an analytical nonlinear gain scheduling (NLGS) approach, which combines the input-output feedback linearization (FBL) and gain scheduling technique, is developed. The stability of the solution is established based on the Reverse Trajectory Method (RTM) to various scheduling points. In addition, the design methodology makes 
use of the formalism of the RTM to maximize the initial guaranteed stability region around an operating point (Genesio et al., 1985). The advantage of the developed approach lies in the fact that we rely upon several exact equivalent models around all operating points. Consequently, the estimated asymptotic stability regions are larger than those obtained through linear approach models.

Thus, trajectory tracking is ensured, not only with better accuracy, but also with improved rapidity and a high degree of stability.

The main goal of this paper is to develop the performance analysis of NLGS control strategies (Charfeddine et al., 2012a; Charfeddine et al., 2012b; Charfeddine et al., 2012c) that ensures trajectory tracking, which involves getting the MPPT of the PV solar powered water pumping system. Two nonlinear polynomial approaches are investigated; continuous and discrete time.

The main contribution of this work is to build the framework of a comparative study to better show that one can preserve all of the performances of the continuous NLGS; despite significant changes in terms of modelling and the fundamental aspects of the continuous and discrete-time systems.

In this research work, a PV system model will be studied. Then, a nonlinear control approach will be presented. Finally, we will give several simulation results to test the robustness and the global performance of the proposed control law.

\section{Materials and methods}

\subsection{Quick description of the system's main components}

In this work, we aim to design an efficient water pumping system that is directly coupled to a solar PV system. The Fig. 1 describes the investigated process.

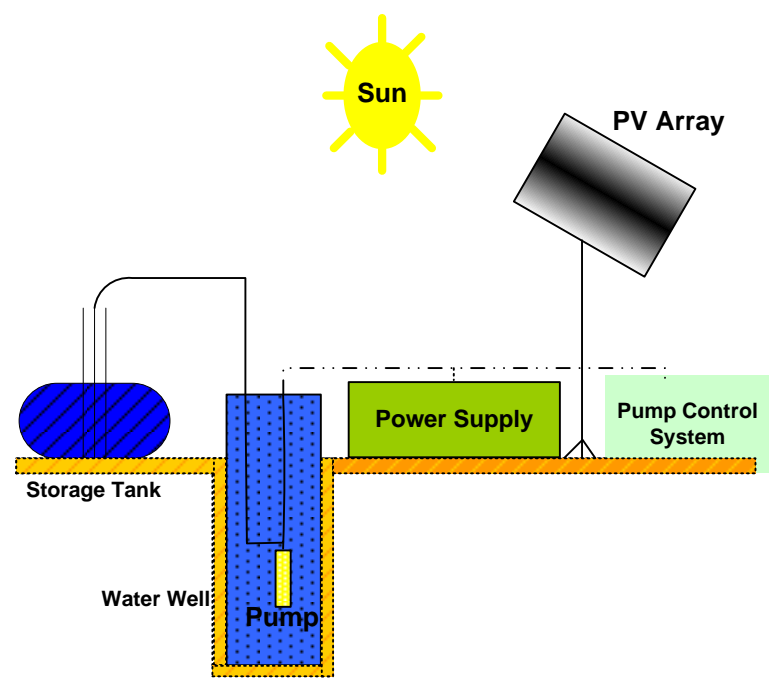

Fig. 1: A schematic of a PV solar powered water pumping system

A photovoltaic system is made up of four main blocks (as shown in Fig. 1). The first block represents the power source (solar panel), the second is a DC-
DC static converter, the third is the load and the fourth is the control system. The main role of the static converter is to make an impedance adaptation, so that the panel produces maximum energy (Mimouni et al., 2004; Andoulsi et al., 1999).

The basic component of the photovoltaic panel is the photovoltaic cell. It is regarded as an ideal current source, as it provides a current $I_{p h}$ that is proportional to the incident light power in parallel with a diode, which is represented by the P-N junction. Therefore, the PV cell can be modeled using the following equations (Mimouni et al., 2004; Andoulsi et al., 1999) (Eq. 1):

$I_{P V}=I_{p h}-I_{d}$

the current $I_{p h}$ is provided by this equation (Eq. 2):

$I_{p h}=\left(I_{c c}+K_{i} \Delta T\right) \frac{E}{E_{n}}$

the expression of the current at the junction level is (Eq. 3):

$I_{d}=I_{S}\left(\exp \left(\frac{V_{p}}{V_{T}}\right)-1\right)$

where $I_{p h}$ and $V_{p}$ are the solar generator output current and voltage, respectively.

The different parameters of the PV cell are summarized in the Table 1.

The DC-DC converter is an interface that allows adapting the PV module and the load, so as to extract the panel's maximum power.

In order to overcome the problems associated with the efficiency of the solar panels and get maximum energy, an MPPT controller will be used to extract the maximum energy whilst taking into account climatic variations (i.e., brightness and temperature). Since 1968 (when the first MPPT control law mode was published (Boehinger, 1968), researchers have developed several optimization techniques. Analogue methods are often simple and cheap. Of these, we may cite the control technique of the generator output voltage, the 'blind' research of the maximum power incrementing the chopper duty cycle ratio, known as the power gradient and modulation through synchronous detection.

MPPT control is a key control for the optimal operation of a PV system. The principle of this control is based on the automatic variation of the duty cycle $\alpha$ by bringing it back to the optimal value to maximize the power delivered by the PV panel.

\subsection{Trajectory tracking importance}

When a photovoltaic panel is fixed to the ground and directed to the south, its energetic efficiency is not constant during the day, whether at the beginning or end of the day. The panel's bad illumination angle decreases power generation performance.

The power production diagram (Mrabti et al., 2010) shows that on a sunny summer's day, the 
Table 1: Parameters of a PV cell

\begin{tabular}{cc}
\hline Thermodynamic potential & $V_{T}=\frac{n K_{B} T}{q}$ \\
\hline PV generator photocurrent proportional to the irradiance level & $I_{p h}$ \\
PV cell reverse saturation current diode, & $I_{S}$ \\
Electron charge. & $q$ \\
Constant of Boltzman's constant & $K_{B}$ \\
Temperature of a solar array $\left({ }^{\circ} \mathrm{K}\right)$, & $T$ \\
The idealistic factor for PN junction & $N$ \\
\hline
\end{tabular}

\subsection{Methodology of synthesis of a trajectory tracking control for the PV solar powered water pumping system}

This section is devoted to the formulation and synthesis of a fuzzy polynomial gain scheduling approach of a PV solar powered water pumping system (Shamma and Athans, 1991; Shamma and Athans, 1992). The proposed technique is based on the methodology of a state feedback control based on a linearizing transformation of the closed loop system.

The proposed solution is based on the use of the concept of asymptotic stability in an initial region of asymptotic stability (Shamma and Athans, 1991; Benhadj et al., 2008). The centre of this region is a stable equilibrium point, which is a point of attraction that can bring any point belonging to its region towards the asymptotic equilibrium. By specifying the desired output trajectory, it is possible to ensure appropriate trajectory tracking by determining the stabilizing control laws of the deviation models calculated in the neighbourhood of the stationary points. Based on the topological considerations, these points are obtained on the desired trajectory (Bacha et al., 2007; Bacha et al., 2008). This will experimentally be applied to a PV solar powered water pumping system.

Establishing the state space equations of the PV system (Andoulsi et al., 2013; Veerachary et al., 2003) (Eq. 4):

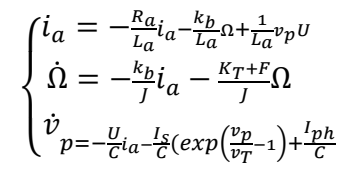

writing the system in the standard form (Eq. 5):

$\left\{\begin{array}{l}\dot{X}=F(X)+G(X) U \\ y=H(X)\end{array}\right.$

where

$$
X=\left[\begin{array}{c}
i_{a} \\
\Omega \\
v_{p}
\end{array}\right]
$$

is the state vector including the motor current, the motor speed and the PV generator voltage.

Calculating the system relative degree $r$ by deriving $y$ until the appearance of the control variable $u$ (Eq. 6):

$$
\dot{y}=\dot{v}_{p}=L_{f} h(X)+U L_{g} h(X)
$$

In the case of continuous system modelling, where $r<n$, the non-linear system dynamics are divided into an input-output external part and an unobservable internal part.

Consequently, the regulation in the neighbourhood of an operating point is now the only objective achieved by the input-output linearizing control.

Let us consider the following variable change $\left(x=X-X_{n}, u=U-U_{n}\right)$; $\quad$ where the operating point state vector is $X_{n}$ and $U_{n}$ is the corresponding control input. Using the development into generalized Taylor series expansions and the Kronecker tensorial product, the model (4). can be readily transformed into a variation polynomial model, expressed by the following equation (Eq. 7):

$\dot{x}=\sum_{i=1}^{q} f_{i} x^{[i]}+\sum_{j=0}^{q}\left(g_{j} x^{[j]}\right)$

where the dimensions of the matrices $f_{i}$ and $g_{j}$ are respectively $\left(n \times n^{i}\right),\left(n \times n^{j}\right)$ and $q$ is a truncation order, $x$ is the variation vector of the state variables and $u$ is the variation control input. The expressions $f_{i}$ and $g_{j}$ are detailed in (Charfeddine et al. 2012a).

Such a model will be used to characterize the feedback linearizing control law, which ensures the regulation around an operating point shall vary along the desired trajectory.

Consequently, it is easy to express the feedback in the following polynomial form (Eq. 8):

$u=\sum_{i=1}^{q} \lambda_{\cdot i} x^{[i]}$

In order to obtain the discrete time model of the system (7), let us consider a suitable selected sampling period $T$ and adopt the following approximations in an interval (Eq. 9):

$\left\{\begin{array}{l}X(t)=\frac{X_{k+1}+X_{k}}{2} \\ \dot{X}(t)=\frac{X_{k+1}-X_{k}}{T}\end{array}\right.$

Hence, the discrete model of the PV solar powered water pumping system can be described by the following polynomial recurrent equation (Eq. 10):

$X_{k+1}=S_{1} X_{k}+S_{2} X_{k}^{[2]}+S_{3} X_{k}^{[3]}+L_{0} U_{k}$

where the matrices $S_{1}, S_{2}$ and $S_{3}$ are deduced by identification (Charfeddine et al. 2012b). 
The polynomial development, truncated to the third order of the PV system around the operating point $\left(X_{n}, U_{n}\right)$, leads to the following polynomial variation model (Eq. 11):

$x_{k+1}=S_{1} x_{k}+S_{2} x_{k}^{[2]}+S_{3} x_{k}^{[3]}+L_{0} u_{k}$

the expression of the diffeomorphism developed in the polynomial form is written as (Eq. 12):

$T\left(x_{k}\right)=T_{1} x_{k}+T_{2} x_{k}^{[2]}+T_{3} x_{k}^{[3]}$

the control input $\mathrm{u}$ is expressed by (Eq. 13):

$u_{k}=\delta_{1} x_{k}+\delta_{2} x_{k}^{[2]}+\delta_{3} x_{k}^{[3]}$

the controlled system (11) should be equivalent to the linear system (Eq. 14):

$z_{k+1}=M z_{k}$

where $z_{k}$ is given by the polynomial transformation (Eq. 15):

$z_{k}=T_{1} x_{k}+T_{2} x_{k}^{[2]}+T_{3} x_{k}^{[3]}$

If we consider an operating point in the desired trajectory given by $\left(X_{n}, U_{n}\right)$, then, according to the previous algebraic development, we can determine the numerical values of the necessary matrices to characterize the local control input and the corresponding nonlinear transformation.

The matrix $\lambda_{1}$ is selected, such that it will be possible to place the poles of the closed system loop with respect to the desired performance.

The matrices $T_{1}, T_{2}, T_{3}, \lambda_{2}$, and $\lambda_{3}$ are detailed in the (Charfeddine et al. 2012c).

The autonomous model is finally described by the following equation (Eqs. 16 and 17):

$x_{k+1}=A_{1} x_{k}+A_{2} x_{k}^{[2]}+A_{3} x_{k}^{[3]}$

where:

$\left\{\begin{array}{l}A_{1}=f_{1}+g_{0} \delta_{1} \\ A_{2}=f_{2}+\left(g_{0} \otimes \delta_{2}\right) \\ A_{3}=f_{3}+\left(g_{0} \otimes \delta_{3}\right)\end{array}\right.$

\section{Results and discussion}

The simulation of the controlled PV system was carried out in a Matlab environment. The numerical parameter values used are shown Table 2.

Table 2: Numerical parameters of the PV solar pumping system

\begin{tabular}{cc} 
& system \\
\hline PV generator: & $I_{p h}=4.4 A, I_{s}=52.75 \mu A, V_{T}=6.73 V$ \\
\hline Capacitor & $\mathrm{C}=400010-6 \mathrm{~F}$ \\
DC motor & $\mathrm{Ra}=1.072 \Omega, \mathrm{La}=0.05 \mathrm{H}, \mathrm{J}=476.10-6, \mathrm{~F}=88.10-$ \\
parameters & $5 \mathrm{pu} \mathrm{KT}=14.10-4 \mathrm{pu}, \mathrm{Kb}=45.10-3 \mathrm{pu}$ \\
\hline
\end{tabular}

The permanent magnet DC motor-pump is characterized by a nominal operating point: $U_{n}=$
$24 \mathrm{~V}$ and $I_{n}=12 \mathrm{~A}, \omega_{n}=2000 \mathrm{rpm}$ and a power $P_{n}=$ $0.3 P U$.

The first step was already carried out to examine the system in the variation model. This study aims to define the best choice of sampling time. According to the Shannon theorem, the value of $\mathrm{T}=0.2 \mathrm{~s}$ offers satisfactory results. Indeed, when comparing the state variables of the systems (7) and (11), it is obvious that they evolve in perfect agreement. This result is shown in Fig. 2. Our objective is now to check the efficiency of the synthesized control inputs described by equations (8) and (13). The results of this study are shown in Figs. 3, 4, and 5. The global performance is satisfactory; indeed, the controlled state variable dynamics are asymptotically stable with rapid transitory response and an accurate steady state regime.

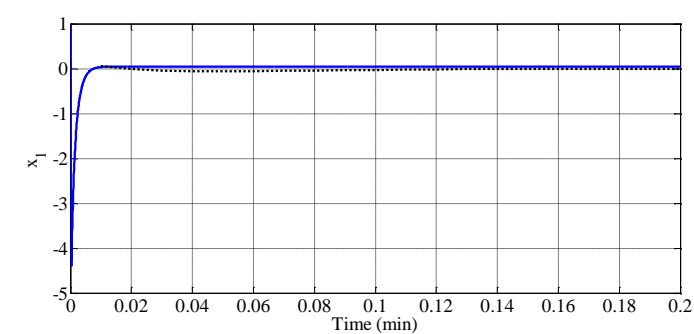

Fig. 2: Dynamics of the variable x1((..): Discrete case, (---) Continuous case)

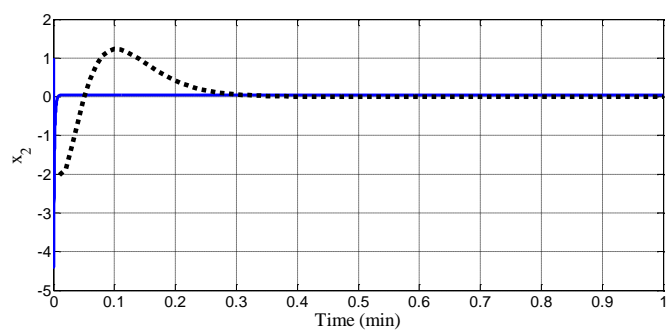

Fig. 3: Dynamics of the variable $x 2$ ((...): Discrete case, (---) Continuous case)

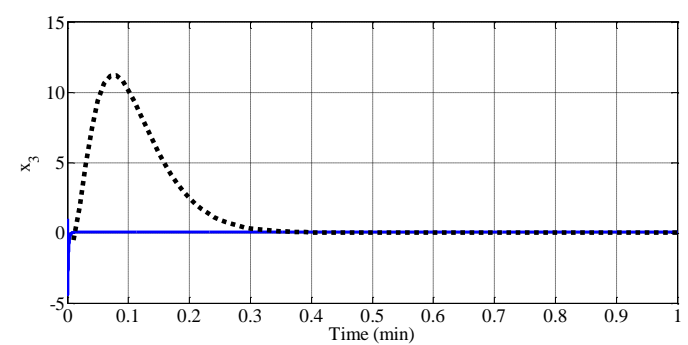

Fig. 4: Dynamics of the variable $\mathrm{x} 3((\ldots)$ : Discrete case, (---) Continuous case)

These figures emphasize the satisfactory dynamic behaviour versus the controlled variable's regulation. Indeed, the simulated continuous and discrete-time variation variables are quickly attracted by the origin and the behaviours observed in these variables do not reveal any inadmissible excess.

However, in terms of speed, the rapidity shown by the continuous variables can be seen. This is due to the assumptions when choosing the truncation order and sampling time. In addition, the feedback 
linearizing technique is more efficient than the polynomial discrete-time technique that we implemented for the discretized model.

The control, as shown in Fig. 5, manages to completely stabilize the system in a few seconds; with regards to the desired set of points. Particularly in the discrete case, the system is stabilized within 2 seconds; however, in the continuous case, it is stabilized in 4 seconds.

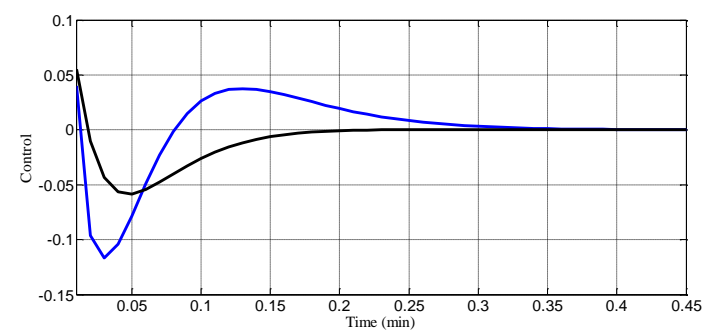

Fig. 5: Control input dynamics law u ((...): Continuous case, (---) Discrete case)

Once the linearizing control law is given, it is possible to apply one of the variable linear control laws. The control's objective is to always maintain the PV generator voltage $\left(\mathrm{y}(\mathrm{t})=V_{p}\right)$ at an optimal value $\left(\mathrm{yd}(\mathrm{t})=V_{p_{0}}\right)$ that corresponds to the point of maximum power. By neglecting the reference variation, this objective will be achieved by choosing the controller $v$, thus ensuring a linear pole placement.

We consider a desired trajectory to be defined by an interval. This trajectory evolves in the form of crenels marking an acceptable physical behaviour in relation to the characteristics and dynamics of the studied photovoltaic system (case increasing sunlight irradiation due to a sandstorm (Salas et al., 2006)) Fig. 6 represents the evolution of the desired trajectory with respect to time.

Fig. 7 represents the evolution of the analytical gain scheduling control law. The dynamics of this control signal are quite satisfactory. In fact, there is no undesirable or unacceptable dynamic excess. We can also see that the response time of the controlled variable is reduced and the tracking error is very small. According to all of these performances, we can say that the developed process control is very interesting.

\section{Conclusion and recommendations}

The synthesis of a discrete-time gain scheduling approach that ensures better trajectory tracking for PV solar pumping system is the main objective of this research work. The PV generator is coupled to a DC motor-pump via a buck converter. A specific output trajectory is derived according to environment characteristics. Indeed, deserted remote regions are particularly characterized by sandstorms, which involve crenels sunlight irradiation behaviour.

The simulation's results are concerned with the evaluation of the transient regime performance of the output variables $\left(V_{p}, I_{a}\right.$ and $\Omega$ ) during the startup process. The curves presented in the previous paragraph highlight the MPPT performance of the studied system.

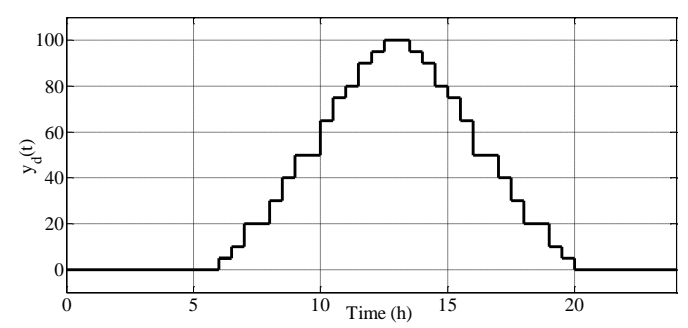

Fig. 6: Desired tracking trajectory

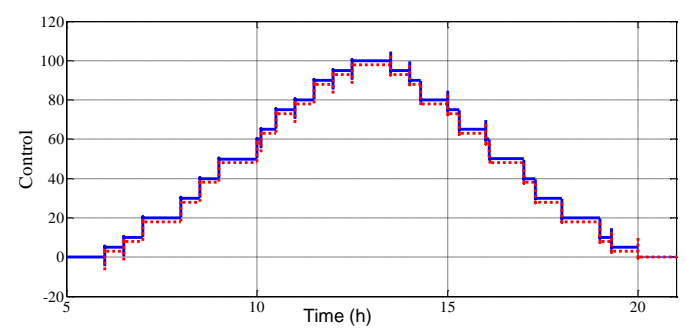

Fig. 7: Control inputs Dynamics ((...): Discrete Time, (---): Continuous Time)

The simulation's results indicate a satisfactory controller performance. Overall system stability was investigated and showed an interesting steady regime behaviour; regardless of the quick dynamics of the output trajectory. This proves that the implemented strategy is satisfactory and that the PV generator voltage is controlled.

The nonlinear control algorithm of the PV system is now developed and its efficiency has been proved.

\section{Acknowledgment}

The authors would like to express their deepest gratitude to the research deanship of Hail University in Saudi Arabia for their sponsorship, financial support and support in undertaking this research work. This research was supported under the university's research deanship local projects funding scheme (project E35- CC).

\section{References}

Andoulsi R, Draou A, Jerbi H, Alghonamy A, and Khiari B (2013). Nonlinear control of a photovoltaic water pumping system. Energy Procedia, 42: 328 - 336.

Andoulsi R, Mami V, Dauphin-Tanguy G, and Annabi M (1999). Modelling and simulation by bond graph technique of a DC motor fed from a photovoltaic source via MPPT boost converter. In the Conference of Particle Accelerator (CSSC'99), IEEE, NY, USA: 4181-4187. Available online at: http://www.wseas.us/e-library/conferences/athens1999/ Papers/259.pdf

Bacha A, Jerbi H, and Benhadj BN (2007). On the synthesis of a combined discrete reversing trajectory method for the asymptotic stability region estimation of nonlinear polynomial systems. In the $13^{\text {th }}$ IEEE IFAC International Conference on Methods, Models in Automation and Robotics, IEEE, Szczecin, Poland: 243-248. 
Bacha A, Jerbi H, and Benhadj BN (2008). Technique of stability domain determination of nonlinear discreet polynomial system. In the $17^{\text {th }}$ Proceedings of the World Congress the International Federation of Automatic Control, Seoul, Korea: 8690-8694.

Bahgat ABG, Helwa NH, Ahmad GE, and El Shenawy ET (2005). Maximum power point tracking controller for PV systems using neural networks. Renewable Energy, 30(8): 1257-1268.

Benhadj BN, Jerbi H, and Bacha A (2008). Technique of stability domain determination for nonlinear discrete polynomial systems. IFAC Proceedings Volumes, 41(2): 8690-8694.

Boehinger AF (1968). Self adaptative DC converter for solar spacecraft power supply. IEEE Transactions on Aerospace and Electronic Systems, 4(1): 102-111.

Charfeddine S and Jerbi H (2012a). Trajectory tracking and disturbance rejection for nonlinear periodic process: a gain scheduling design. International Review on Modelling and Simulations, 51(2): 1075-1083.

Charfeddine S and Jerbi H (2012b). A Survey of nonlinear gain scheduling design control of continuous and discrete time systems. International Journal of Modelling Identification and Control, 19(3): 203-216.

Charfeddine S and Jerbi H (2012c). Synthesis of a nonlinear control system for disturbance rejection in periodic process. In the $9^{\text {th }}$ International Multi-Conference on Systems Signals and Devices (SSD'12), IEEE, Chemnitz, Germany: 1-6. https://doi.org/10.1109/SSD.2012.6197942

Cocconi A and Rippel W (1990). Lectures from GM Sun racer Case History, Lecture 3-1: The Sun racer Power Systems', Number M-101, Society of Automotive Engineers, Warrendale, USA.

El Ouariachi ML, Mrabti T, Tidahf B, Kassmi Ka, and Kassmi K (2009). Regulation of the electric power provided by the panels of the photovoltaic system. International Journal of Physical Sciences, 4(5): 294-309.

Esram T and Chapman PL (2007). Comparison of photovoltaic array maximum power point tracking techniques. IEEE Transactions on Energy Conversion, 22(2): 439-449.

Genesio R, Tartaglia M, and Vicino A (1985). On the estimation of asymptotic stability regions: State of art and new proposals. IEEE Transactions on Automatic Control, 30(8): 747-755.
Hussein KH, Muta I, Hoshino T, and Osakada M (1995). Maximum photovoltaic power tracking: an algorithm for rapidly changing atmospheric conditions. IEEE ProceedingsGeneration, Transmission and Distribution, 142(1): 59-64.

Mimouni MF, Mansouri MN, Benghanem B, and Annabi M (2004). Vectorial command of an asynchronous motor fed by photovoltaic generator. Renewable Energy, 29(3): 433-442.

Mrabti T, El Ouariachi M, Kassmi K, and Tidhaf B (2010). Characterization and modeling of the optimal performances of the marketed photovoltaic panels. Moroccan Journal of Condenser Mater, 12(1): 7-13.

Mrabti T, El Ouariachi M, Yaden M, Kassmi Ka, and Kassmi K (2010). Characterization and modeling of electrical performance of the photovoltaic panels and system. Journal of Electrical Engineering: Theory and Application, 1(2): 100-110.

Salameh ZM, Dagher F, and Lynch WA (1991). Step down maximum power point tracker for photovoltaic systems. Solar Energy, 46(5): 279-282.

Salas V, Olias E, Barrado A, and Lazaro A (2006). Review of the maximum power point tracking algorithms for stand-slone photovoltaic systems. Solar Energy Materials and Solar Cells, 90(11): 1555-1578.

Shamma JS and Athans M (1991). Guaranteed properties of gain scheduled control for linear parameter-varying plants. Automatica, 27(3): 559-564.

Shamma JS and Athans M (1992). Gain scheduling: potential hazards and possible remedies. IEEE Control Systems, 12(3): 101-107.

Shraif MF (2002). Optimisation et mesure de chaîne de conversion d'énergie photovoltaïque en énergie électrique. Ph.D. Dissertation, Université Paul Sabatier, Toulouse, France.

Swrup T and Ansari A (2012). Maximum power point tracking method for multiple photovoltaic systems. Research Journal of Chemical Sciences, 2(12): 69-77.

Veerachary M, Senjyu T, and Uezato K (2003). Neural-Network based maximum power point tracking of coupled inductor interleaved boost converter supplied PV system using fuzzy controller. IEEE Transactions on Industrial Electronics, 50(4): 749-758. 